\title{
AlgaePath: comprehensive analysis of metabolic pathways using transcript abundance data from next-generation sequencing in green algae
}

\author{
Han-Qin Zheng ${ }^{2}$, Yi-Fan Chiang-Hsieh ${ }^{1}$, Chia-Hung Chien ${ }^{1}$, Bo-Kai Justin Hsu' ${ }^{4}$ Tsung-Lin Liu²,
} Ching-Nen Nathan Chen ${ }^{3^{*}}$ and Wen-Chi Chang ${ }^{1,2^{*}}$

\begin{abstract}
Background: Algae are important non-vascular plants that have many research applications, including high species diversity, biofuel sources, and adsorption of heavy metals and, following processing, are used as ingredients in health supplements. The increasing availability of next-generation sequencing (NGS) data for algae genomes and transcriptomes has made the development of an integrated resource for retrieving gene expression data and metabolic pathway essential for functional analysis and systems biology. In a currently available resource, gene expression profiles and biological pathways are displayed separately, making it impossible to easily search current databases to identify the cellular response mechanisms. Therefore, in this work the novel AlgaePath database was developed to retrieve transcript abundance profiles efficiently under various conditions in numerous metabolic pathways.

Description: AlgaePath is a web-based database that integrates gene information, biological pathways, and NGS datasets for the green algae Chlamydomonas reinhardtii and Neodesmus sp. UTEX 2219-4. Users can search this database to identify transcript abundance profiles and pathway information using five query pages (Gene Search, Pathway Search, Differentially Expressed Genes (DEGs) Search, Gene Group Analysis, and Co-expression Analysis). The transcript abundance data of 45 and four samples from C. reinhardtii and Neodesmus sp. UTEX 2219-4, respectively, can be obtained directly on pathway maps. Genes that are differentially expressed between two conditions can be identified using Folds Search. The Gene Group Analysis page includes a pathway enrichment analysis, and can be used to easily compare the transcript abundance profiles of functionally related genes on a map. Finally, the Co-expression Analysis page can be used to search for co-expressed transcripts of a target gene. The results of the searches will provide a valuable reference for designing further experiments and for elucidating critical mechanisms from high-throughput data.
\end{abstract}

Conclusions: AlgaePath is an effective interface that can be used to clarify the transcript response mechanisms in different metabolic pathways under various conditions. Importantly, AlgaePath can be mined to identify critical mechanisms based on high-throughput sequencing. To our knowledge, AlgaePath is the most comprehensive resource for integrating numerous databases and analysis tools in algae. The system can be accessed freely online at http://algaepath.itps.ncku.edu.tw.

Keywords: Algae, Pathway, Next-generation sequencing, Systems biology, Gene expression

\footnotetext{
*Correspondence: nathanc@mail.nsysu.edu.tw; sarah321@mail.ncku.edu.tw

${ }^{3}$ Institute of Marine Biology, National Sun Yat-sen University, Kaohsiung

80424, Taiwan

${ }^{1}$ Institute of Tropical Plant Sciences, National Cheng Kung University, Tainan

70101, Taiwan

Full list of author information is available at the end of the article
}

C Biomed Central (c) 2014 Zheng et al.; licensee BioMed Central Ltd. This is an Open Access article distributed under the terms of the Creative Commons Attribution License (http://creativecommons.org/licenses/by/2.0), which permits unrestricted use, distribution, and reproduction in any medium, provided the original work is properly credited. The Creative Commons Public Domain Dedication waiver (http://creativecommons.org/publicdomain/zero/1.0/) applies to the data made available in this article, unless otherwise stated. 


\section{Background}

The global energy crisis poses a major threat to human survival. Gradual depletion of non-renewable energy resources has increased energy costs and pollution. To remedy this situation, renewable energy sources such as biomass have been developed. Biomass is gathered from various plants (including crops or peas) and used to generate bio-alcohol or bio-fuel [1,2]. However, using crops as a biomass source may crowd out food, especially in poor regions of the world [3]. Thus, an alternative species with less land requirement and high oil production needs to be identified. Algae are highly efficient in oil production, and are a promising biofuel source [4]. In addition to their possible use for biofuel, algae have many research applications [5]. For instance, because of its high species diversity, algae is an excellent model for evolution studies. The diversity of algae has been attributed not only to the evolution force, but also to horizontal gene transfer [6]. Allochthonous genes can increase species diversity and organism competition [6]. Further, because algae are capable of absorbing heavy metals, they can be used to remove pollution in waste water [7-11]. Currently, algae are the most important non-vascular model plants that are used for research.c Because of their diverse applications, numerous studies have investigated the functional genomics of algae. Therefore, with the increasing availability of algae genome and transcriptome data from nextgeneration sequencing, an integrated resource for the retrieval of gene expression data with metabolic pathways is essential for functional analysis in algae. Further, understanding cellular response mechanisms under different environments is a high priority in systems biology.

Chlamydomonas reinhardtii is a single-celled green algae, which is distributed worldwide in soil and water. $C$. reinhardtii is used mainly as a model organism when addressing fundamental issues such as photosynthesis, cellular movement, abiotic response mechanisms, and regulation of flagellar mechanism. This model organism is also an important model for non-vascular plant research and information on the genomics and transcript abundance of $C$. reinhardtii is available in public resources. Neodesmus sp. UTEX 2219-4 is a species of green microalgae, which was isolated from collection number UTEX 2219 from the University of Texas at Austin (UTEX) and identified subsequently as genus Neodesmus [12]. Wang et al. [12] indicated that oil bodies were accumulated significantly under nitrogen starvation and osmotic stress in Neodesmus sp. UTEX 2219-4. Therefore, this strain was identified as having a high potential for biofuel applications. This study analyzed the transcriptome deep sequencing data that was obtained from four samples under various conditions.

Increasing amounts of next generation sequencing (NGS) data have become available in recent years and algal biochemistry and biology have attracted growing interest for their potential in developing renewable biofuel. In addition, RNA-seq technology has been used widely to identify algal cellular physiology and metabolism under various types of abiotic stress and/or nutrient deficiency [13-18]. For example, a previous study investigated cellular response mechanisms by characterizing the $C$. reinhardtii transcriptome under nutrient-replete and sulfur-depleted conditions [16]. Based on Roche 454 and Illumina sequencing, Miller et al. [17] investigated diversion of metabolism and transcript abundance during nitrogen repletion and deprivation in C. reinhardtii. Consequently, the increasing use of NGS has made the integration of high-throughput data from different experiments a priority concern. Genevestigator [19] is a high performance platform that integrates many public microarray experiments and visualizes gene expression data in different biological contexts. However, although nine important higher plants have been collected in the Genevestigator database, none of them are non-vascular plants. The Bio-Analytic Resource for Plant Biology (BAR) [20] also integrates numerous microarray data and provides expression profile similarity rankings of homologous genes in plant species; however, BAR cannot access algae-related information. BioCyc [21] is a collection of databases that provides the metabolic pathways of sequenced organisms including C. reinhardtii, but no gene expression data have been integrated into the collection. To our knowledge, the Algal Functional Annotation Tool (AFAT) is the first database in which algae gene expression data and metabolic pathways have been collected [22]. AFAT provides an integrated data-mining environment for algal genomics by integrating multiple annotation databases into a centralized system. Unfortunately, the metabolic pathways and gene expression profiles are displayed in separate windows in AFAT, making it difficult for users to understand the variations in cellular responses under different conditions. Additionally, the complex IDs and datasets in AFAT can often confuse users who want to search for interesting genes and pathways. For gene expression analysis, the AFAT website provides only an expression similarity search and the result page outputs only a Gene ID. As a result, it is relatively difficult to compare the variations of gene expression under different conditions using AFAT tools. Surprisingly, there are currently no other databases or tools that can be used to combine gene expression data with metabolic pathways in algae for systems biology, thereby necessitating the development of additional databases and related tools.

In this work, the novel AlgaePath database, which allows the efficient retrieval of cellular response data from different conditions, was developed. AlgaePath integrates various high-throughput datasets of C. reinhardtii and the stressinduced oil accumulation dataset of Neodesmus sp. UTEX 2219-4. AlgaePath can be used to identify transcript 
abundance profiles and to compare variations among different conditions in a pathway map through five query functions (Gene Search, Pathway Search, Differentially Expressed Genes (DEGs) Search, Gene Group Analysis, and Co-expression Analysis). The novel database can be accessed freely online at http://AlgaePath.itps.ncku.edu.tw.

\section{Construction and content}

AlgaePath is a web-based database, which includes a highthroughput experimental dataset and information for C. reinhardtii and Neodesmus sp. UTEX 2219-4. The flowchart of AlgaePath is displayed in Figure 1. The system runs on an Apache web server on a Linux operating system. All the data are stored in a MySQL database and displayed in web pages, which are written in the PHP, Perl, and HTML programming languages. Details of the AlgaePath data and structure are described below.

\section{Transcriptome data of Neodesmus sp. UTEX 2219-4}

The Neodesmus sp. UTEX 2219-4 transcriptome was sequenced on a Roche 454 system. The transcriptome data were obtained under four conditions (normal, nitrogen starvation, sorbitol stress, and salt stress) and have been integrated into the AlgaePath database. The sequence data processing and analysis have not been published (unpublished data in Dr. Ching-Nen Nathan Chen's lab). The assembled sequences were annotated based on their similarity to sequences in the NCBI non-redundant (nr) database and the UniProt UniRef50 protein database. Currently, there are 20,698 Neodesmus $s p$. UTEX 2219-4 transcripts (including isotigs, contigs, and singletons) in the AlgaePath database.

\section{Transcriptome data of $C$. reinhardtii}

The $C$. reinhardtii reference sequences and their annotation were downloaded from Phytozome (http://www.phytozome.net/, v5.3.1 of Chlamydomonas annotations) [23]. A total of 19,529 transcripts with annotations from Pfam $(10,122)$ [24], PANTHER $(8,905)$ [25], NCBI eukaryotic orthologous groups (KOG) $(5,623)$ [26], KEGG $(2,724$ KEGG ortholog), and Arabidopsis homologous genes $(9,106)$ were obtained. The RNA-seq transcriptome data were accessed from the Gene Expression Omnibus (GEO). In this study, the GSE17970, GSE24367, GSE25622, GSE33927, GSE33548, GSE34826, and GSE35305 datasets were used [13-18]. The conditions used for RNA-seq transcripts are sulfur depletion, nitrogen deprivation, mineral nutrient treatment, various concentration of carbon dioxide $\left(\mathrm{CO}_{2}\right)$, oxidative stress, and Fe deprivation, including 45 samples. Adaptors were trimmed from all the sequences in the different samples and then the reads were mapped back to the reference sequences. The Bowtie2 [27] and BLAT [28] programs were used with their default parameters to map the Illumina sequence reads (Solexa) and the 454 pyro-sequence reads separately to the reference sequences. Finally, the expression level of each of the transcripts was calculated using reads per kilobase per million reads (RPKM).

\section{Normalization of expression data of each transcripts and identification differentially expressed genes}

Because different transcriptome datasets were used, Deseq $R$ package was applied to normalize various algae raw data and identify differentially expressed genes (DEGs) depending on negative binomial distribution. DEGs based on 17 comparison groups (see Additional file 1: Table S1) were pre-run and saved in AlgaePath database. However, there is the other option in AlgaePath web interface. Various samples could be selected to compare gene expression level, and identify DEGs based on expression fold change. Furthermore, Giorgi et al. indicated that Variance-Stabilizing Transformated (VST) RNA-seq data brings RNA-seq samples hierarchically closer to microarrays than RPKM normalization or raw counts when reconstructing co-expression networks [4]. Therefore, VarianceStabilizing Transformation (VST) method was used to transform DEseq pre-normalized data. The VST was also performed by Deseq R package [29]. The VST normalized data were than used to analyze co-expression genes.

\section{Analysis of co-expressed genes}

For the co-expression analysis, all the samples (excluding mutant samples) were divided into six categories (all conditions (GSE17970, GSE24367, GSE25622, GSE33927, GSE33548, GSE34826, and GSE35305), nitrogen deprivation (GSE24367), mineral nutrient treatment (GSE17970, GSE25622), carbon dioxide treatment (GSE33927), oxidative stress (GSE34826), and iron deprivation (GSE35305)). Based on Pearson's correlation coefficient (PCC), the similarity of expression patterns across various samples in a category was measured, which represents the co-expressed level between a pair of genes. The co-expressed genes were then calculated, depending on the different categories. Next, the gene-pairs with PCC values ( $\mathrm{r}$ ) between -0.5 and 0.5 were removed from the co-expression data. Finally, the 100 best positive and 100 best negative correlations of a transcript were stored in the AlgaePath database. The PCC values were calculated as:

$$
\mathrm{r}(\text { correlation of a gene pair })=\frac{\sum_{i=1}^{n}\left(X_{i}-\bar{X}\right)\left(Y_{i}-\bar{Y}\right)}{\sqrt{\sum_{i=1}^{n}\left(X_{i}-\bar{X}\right)^{2}} \sqrt{\sum_{i=1}^{n}\left(Y_{i}-\bar{Y}\right)^{2}}}, \text { where }
$$

$\overline{\mathrm{X}}$ and $\overline{\mathrm{Y}}$ denote the average expression values of $X$ and $Y$ genes under all conditions in each category, respectively $n$ represents the total number of samples in each category. 


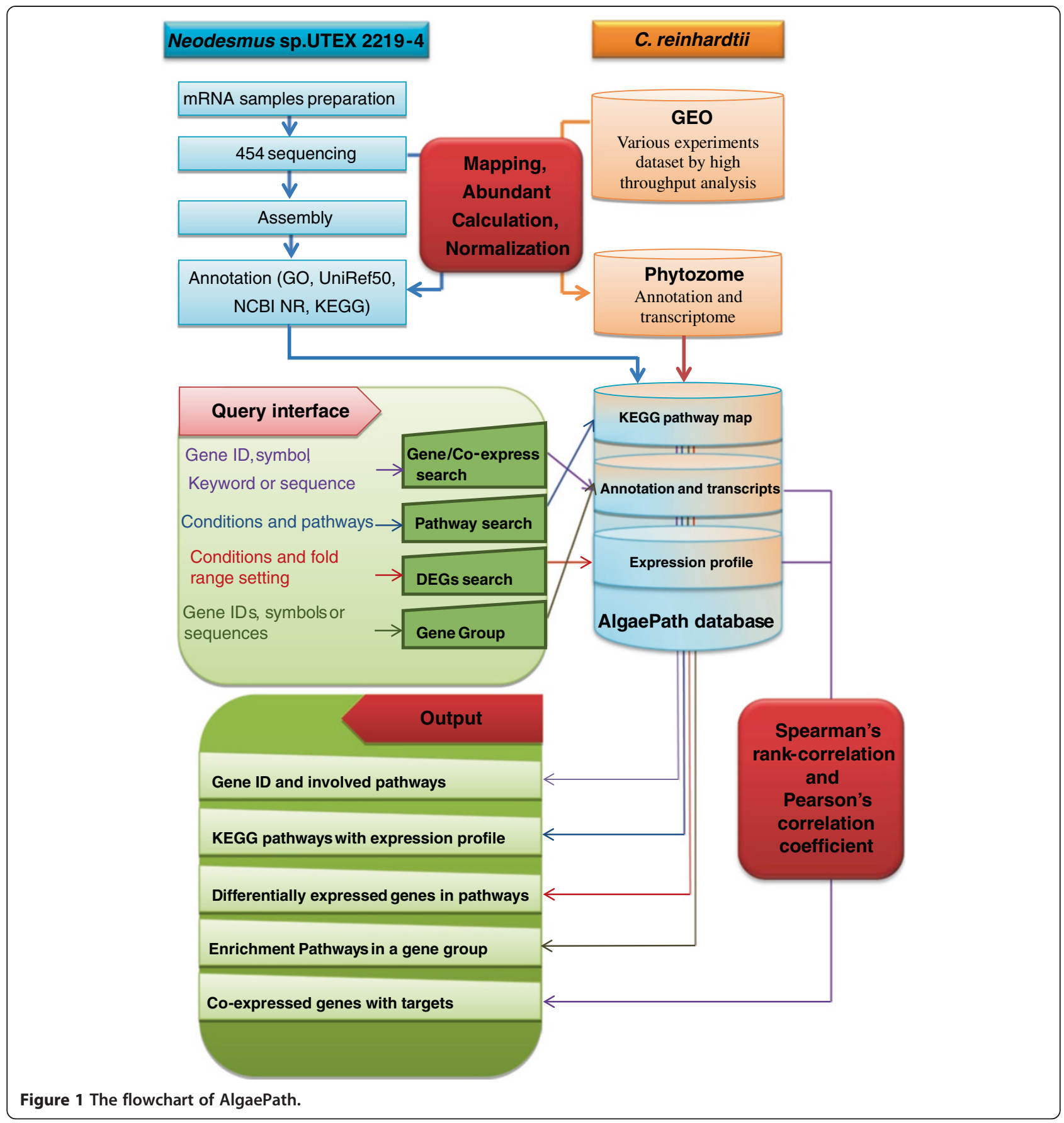

Additionally, The Spearman's rank-correlation is less sensitive than the Pearson correlation to strong outliers. AlgaePath also provide the co-expression results using Spearman's correlation. Users can identify co-expression genes based on different statistics methods.

Pathways identification of each transcript and gene group analysis.

KEGG genes and KEGG orthology (KO) were downloaded from the KEGG database (Release 2013) [30].
The KO IDs were mapped to transcripts annotated in AlgaePath. Related pathways of each transcript were then identified using the KEGG pathway reconstructed tool. For gene group analysis, pathway enrichment in a group of gene sets was analyzed using the hypergeometric distribution method [31]. The number of transcripts involved in each pathway was calculated as the abundance pathways in a group of gene sets. Pathway enrichment was then verified based on the p-value of each pathway 
following the hypergeometric distribution method as follows:

$$
\mathrm{P}_{j}=\sum_{i=x}^{n} \frac{\left(\begin{array}{c}
M \\
i
\end{array}\right)\left(\begin{array}{c}
N-M \\
n-i
\end{array}\right)}{\left(\begin{array}{c}
N \\
n
\end{array}\right)}
$$

where, $i$ denotes the number of transcripts in the gene group involved in the $j$ pathway; $n$ is the total number of transcripts in the gene group annotated in KEGG; $M$ is the number of all $C$. reinhardtii transcripts involved in the $j$ pathway; and $N$ is the total number of all Ch. reinhardtii transcripts annotated in KEGG.

\section{Utility and discussion}

\section{Web interface of AlgaePath}

Two species (C. reinhardtii and Neodesmus sp. UTEX 2219-4) are displayed on the AlgaePath home page (Figure 2). By clicking on either of the species (for example C. reinhardtii), five transcriptome analysis functions are displayed; namely, Gene Search, Pathway Search, Differentially Expressed Genes (DEGs) Search, Gene Group Analysis, and Co-expression Analysis. Tutorial and Browse options are displayed at the top of the home page for each species. On the Gene Search page, a gene symbol, keyword, gene ID from various databases (Pfam, PATHER, KEGG K ENTRY, and KOG), DNA sequence, or protein sequence can be input as a query. Gene Search uses the query to retrieve the gene expression profiles in a specific pathway under different conditions (Figure 3). The Pathway Search page allows users to first select the stress conditions of interest. This opens the pathway browser in which either one of the listed pathways can be selected or a pathway search can be performed using a keyword. The algae genes (marked in green) involved in the pathway are then displayed in a pathway figure. Clicking on an identified gene opens a link to the KEGG ortholog information for that gene. Expression level profiles under various conditions and information about the gene are also displayed. The DEGs Search page has two options: "Analyzing by Deseq $\mathrm{R}$ package" and "Fold change search". DEGs from 17

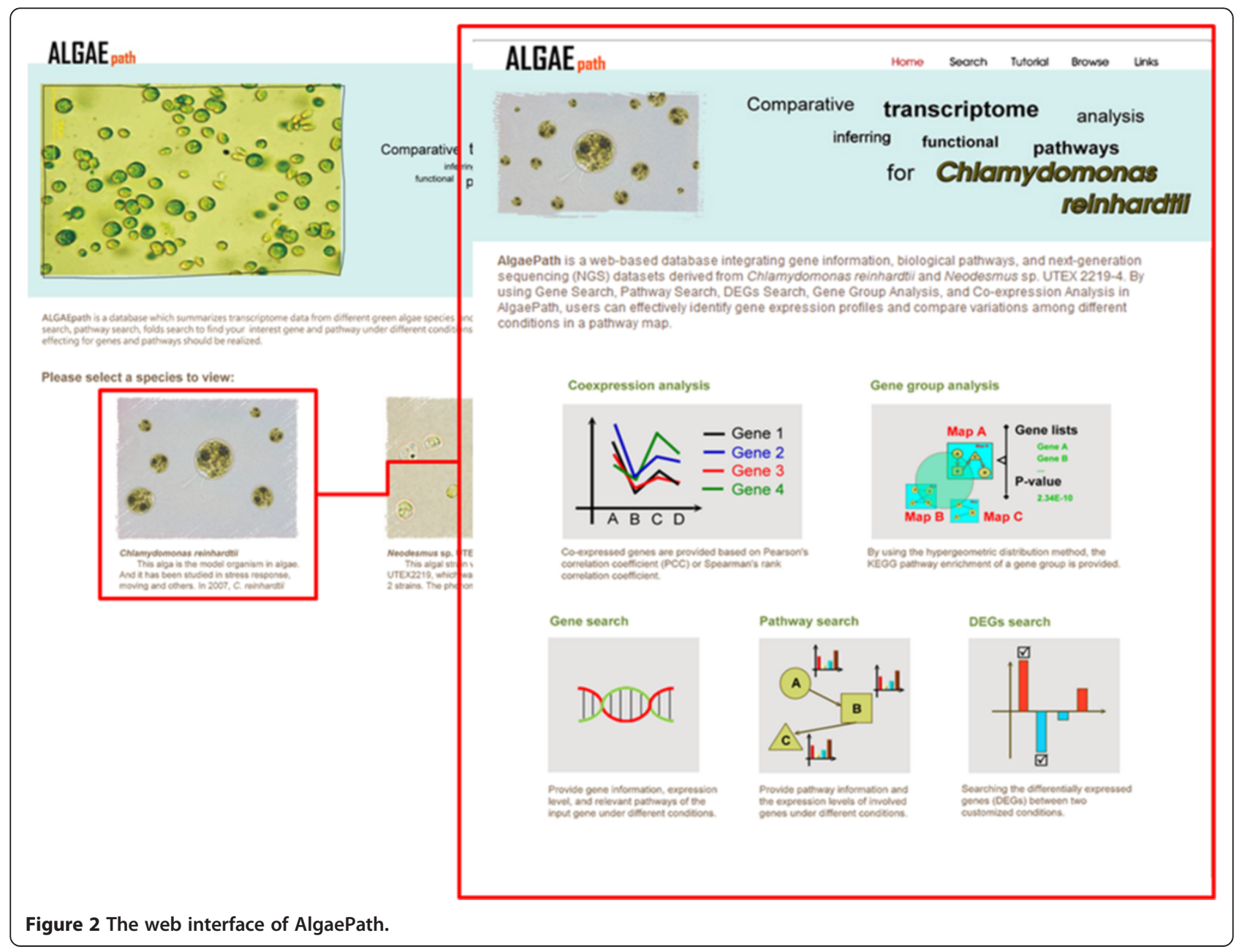




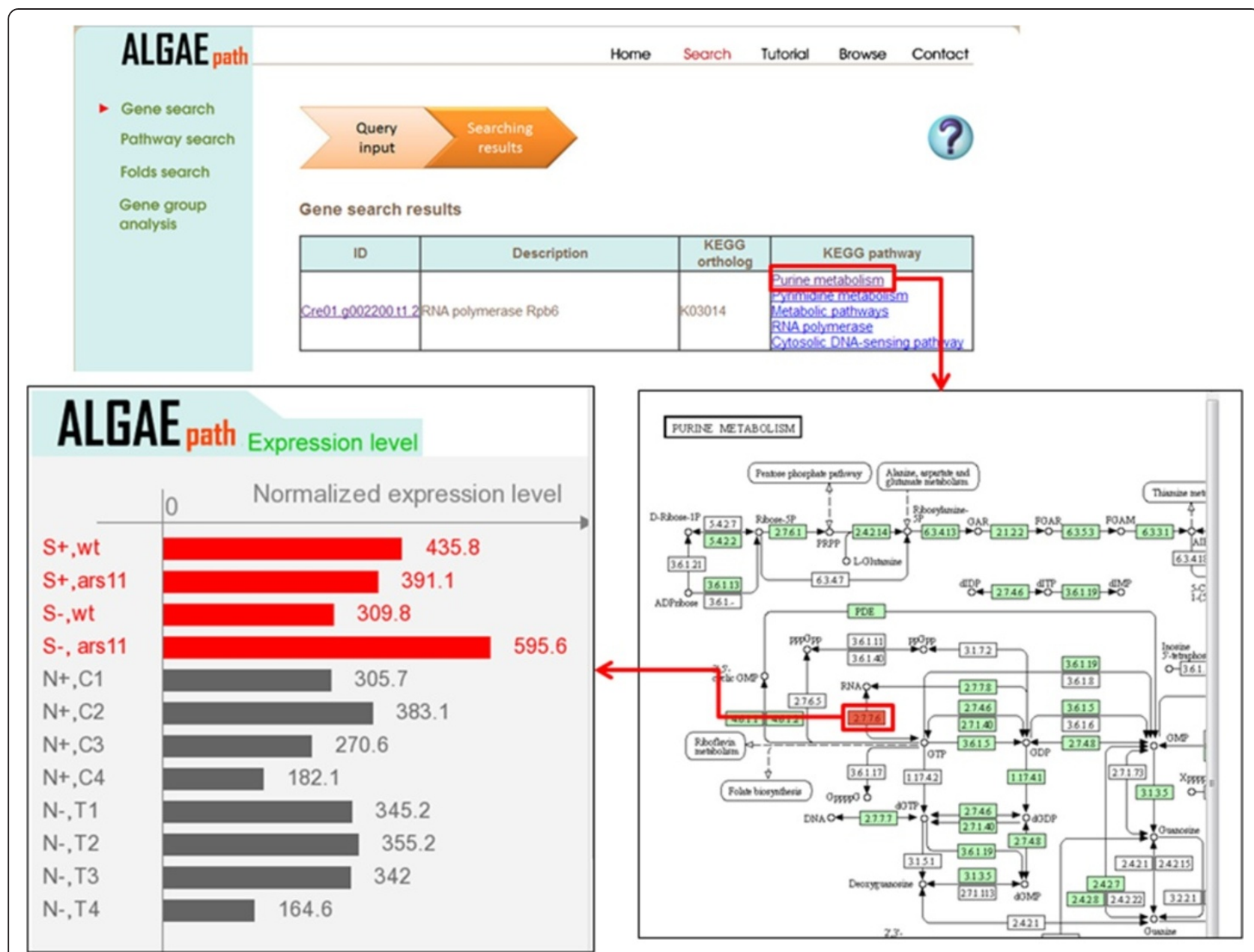

Figure 3 The output result of "Gene Search" in AlgaePath. The input gene is marked in red background, and the gene expression levels under different conditions are displayed in a popup window.

comparison groups can be identified by using "Analyzing by Deseq R package", respectively. "Fold change search" can be used to compare differentially expressed genes between two conditions that can be selected by the user. The fold range can be chosen and/or keywords can be used to limit the number of results. The search results pages are similar either use "Analyzing by Deseq R package" or "Fold change search". The numbers of genes within the chosen fold range or p-value are displayed both as a graph and in a table. Detailed information about the genes and pathways with a particular fold change can be accessed by clicking on the gene numbers in the table (Figure 4). The Gene Group Analysis page provides a tool for users to perform pathway enrichment analysis for a group of input genes. Pathways related to the user-selected group of genes are listed in a table which also displays a hit percentage and $\mathrm{p}$-value (Figure 5). The pathway view button can be used to display the transcript abundance levels of these genes under numerous conditions. The Co-expression Analysis page can be used to retrieve gene information and expression profiles of genes that have similar expression profiles (based on PCC or Spearman's correlation) to a user-input gene. First, the condition of interest and the gene of interest are selected. Then co-expressed genes under a particular experiment are identified and top ten correlated genes for each query gene are displayed. The functional related genes with similar expression profiles across different experimental conditions can be accessed directly (Figure 6). Up to 100 positive and negative correlations can be accessed from different pages. Co-expression analysis can also be accessed from the Gene Search page.

\section{Comparative analysis of gene expression profiles under various conditions}

Comparative analysis of gene expression under various conditions can help biologists identify critical genes involved in changes of interest. For example, the feasibility of using algae in biofuel production has been studied [4] and scientists have attempted to overexpress acetylCoA carboxylase (a substrate for fatty acid production) 


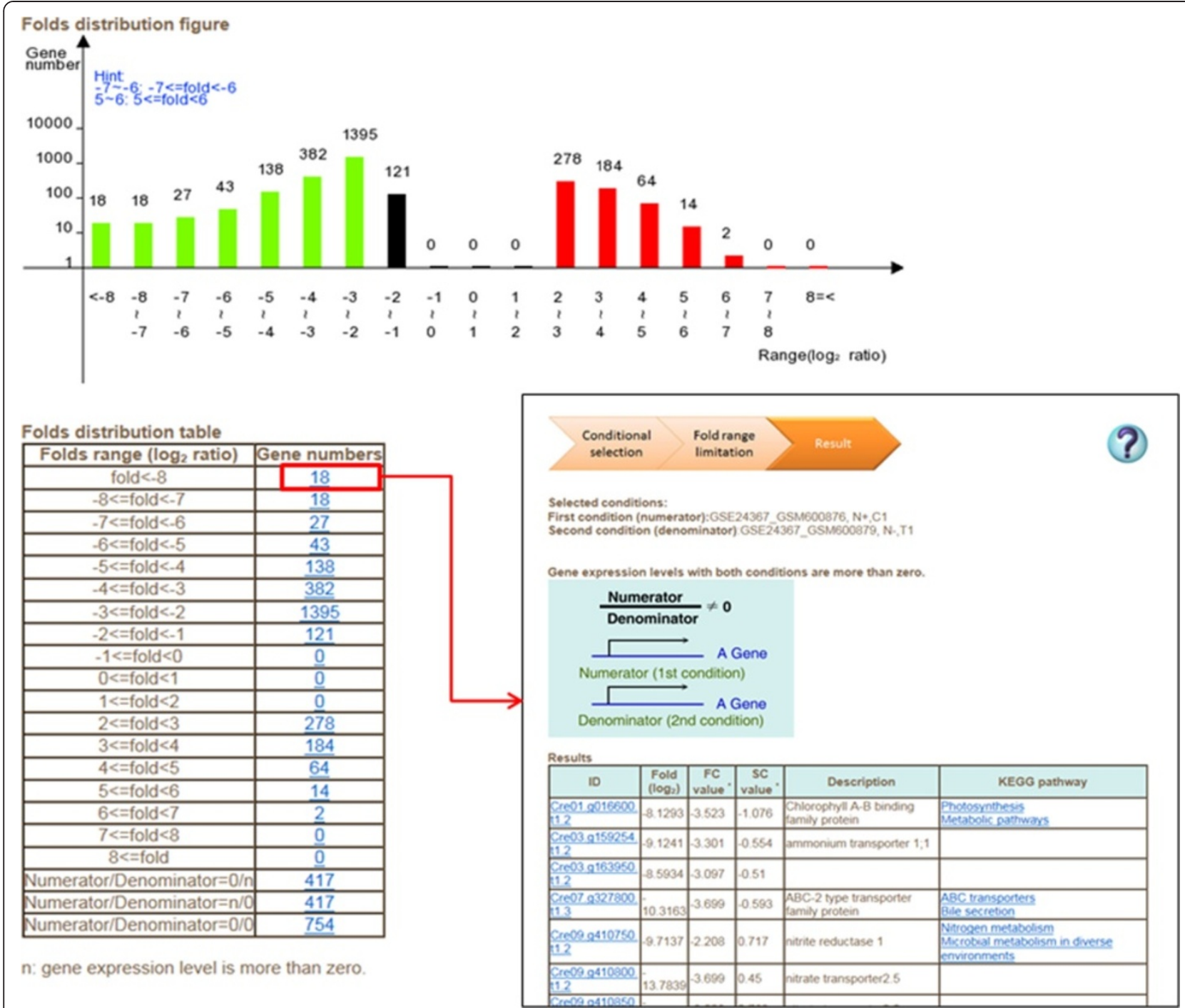

Figure 4 The output results of "Differentially Expressed Genes (DEGs) Search" in AlgaePath. The statistics numbers of genes in particular folds ranges are listed in a table. The further information about those genes could be retrieved by clicking the gene numbers on the web page.

in microalgae to improve oil production [29]. Unfortunately, until now, the performance of algae in oil production has been insignificant [32]. Rismani-Yazdi et al. [33] recently identified some important metabolic pathways related to microalgae-based biofuel feedstock by comparing transcriptome sequencing data under four different conditions. Numerous NGS transcriptome datasets are currently available in public resources and they need to be integrated so that important checkpoints can be identified in biological mechanisms. Importantly, in the AlgaePath database, users can easily investigate variations in transcript abundance under various conditions in a specific pathway based on user selection. All output result pages are displayed in a figure and in a downloadable table, as shown in Figure 3. The user-friendly interface can help users determine efficiently whether their genes of interest undergo significant changes in expression under different conditions. In this sense, AlgaePath is similar to the other resources that have been developed for higher plants (e.g. Genevestigator and the BAR database). Importantly, the biological pathways in AlgaePath are thoroughly combined with the transcript abundance data. Many important phenotypic changes that cause serious biological reactions are affected by more than one gene; therefore, researchers need to determine which genes have expression patterns that are similar to their target gene. Such information can help biologists clarify the mechanisms that they are interested in. Because co-expression analysis is very important in systems biology research, the ability to study the co-expression of genes in the six categories available in AlgaePath will be highly effective. The six categories were designed to provide users with a variety of options for data mining because many 


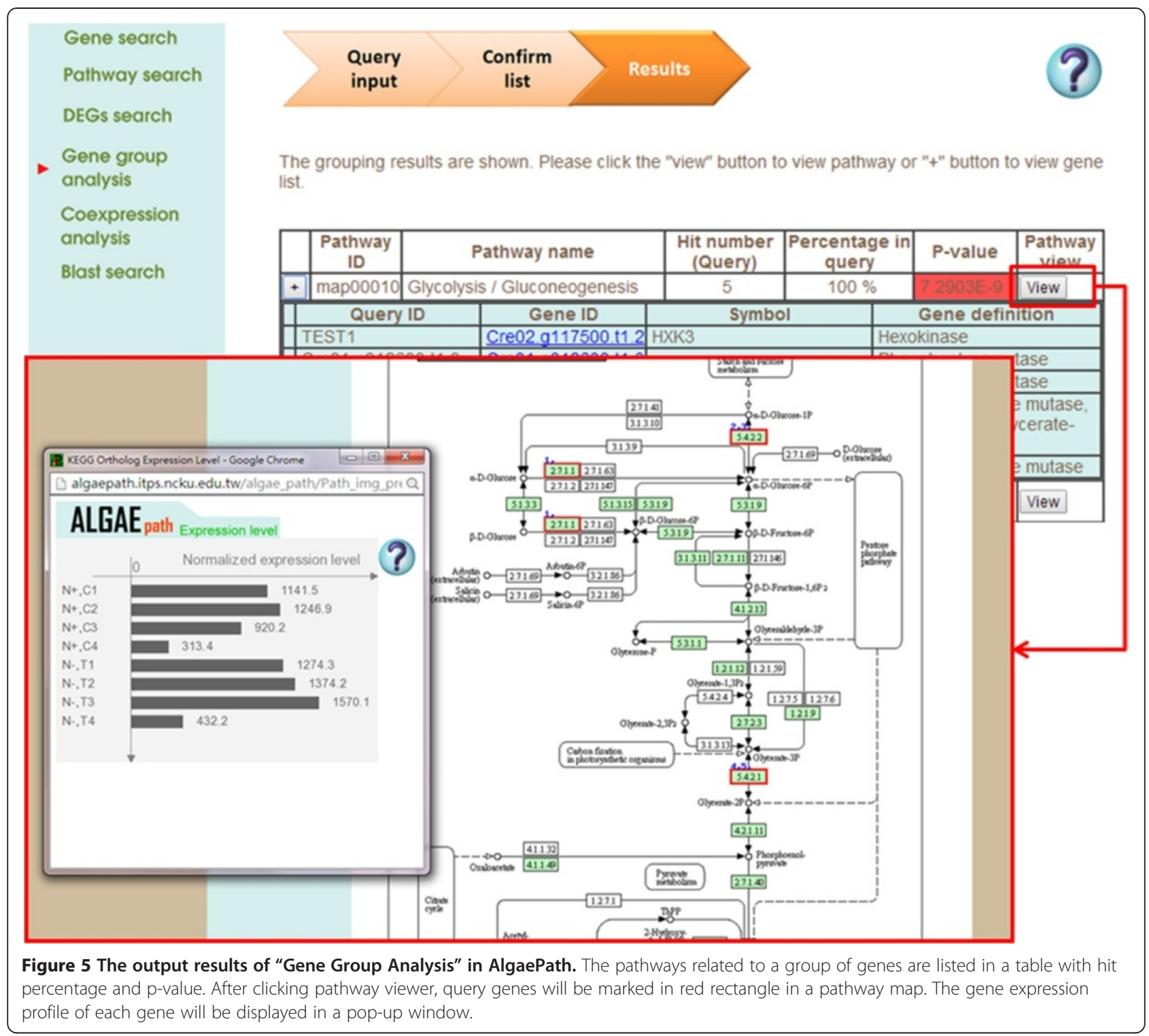

genes are co-expressed only under specific conditions. The AlgaePath system will provide users a multifunctional analysis platform for algae-related research.

\section{Case study: identification of critical genes during nitrogen starvation and sulfur depletion}

The accumulation of oil bodies in C. reinhardtii under nitrogen starvation conditions was reported previously [34]. Several up-regulated and down-regulated genes in lipid biosynthesis pathway under nitrogen starvation conditions were identified using the AlgaePath database [17]. For instance, the diacylglycerol O-acyltransferase (DGAT, Cre12.g557750.t1.3), fatA acyl-ACP thioesterase (Cre06. g256750.t1.2), and plant stearoyl-acyl-carrier-protein desaturase family protein (Cre17.g701700.t1.2 and g17011.t1) genes were found to be significantly up-regulated under nitrogen starvation using the DEGs Search tool in AlgaePath. In addition, the variation of the expression profiles of genes involved in the fatty acid biosynthesis pathway were identified easily using Pathway Search. Sulfur is essential in the synthesis of proteins, lipids, and various metabolites. Because most organisms, including $C$. reinhardtii, have limited sulfur storage abilities, the continuous uptake of sulfur from the surrounding environment is critical to their survival. Based on microarray experiments, Zhang et al. [35] identified the responses of numerous genes to sulfur depletion. The identified genes were involved in sulfur metabolism, photosynthesis, carbon metabolism, respiration alternative electron transfer pathways/ATPase/ transporters, oxidative stress, chaperones, proteolysis, signal transduction, and transcription, and, in particular, several genes related to sulfur metabolism or biosynthesis 


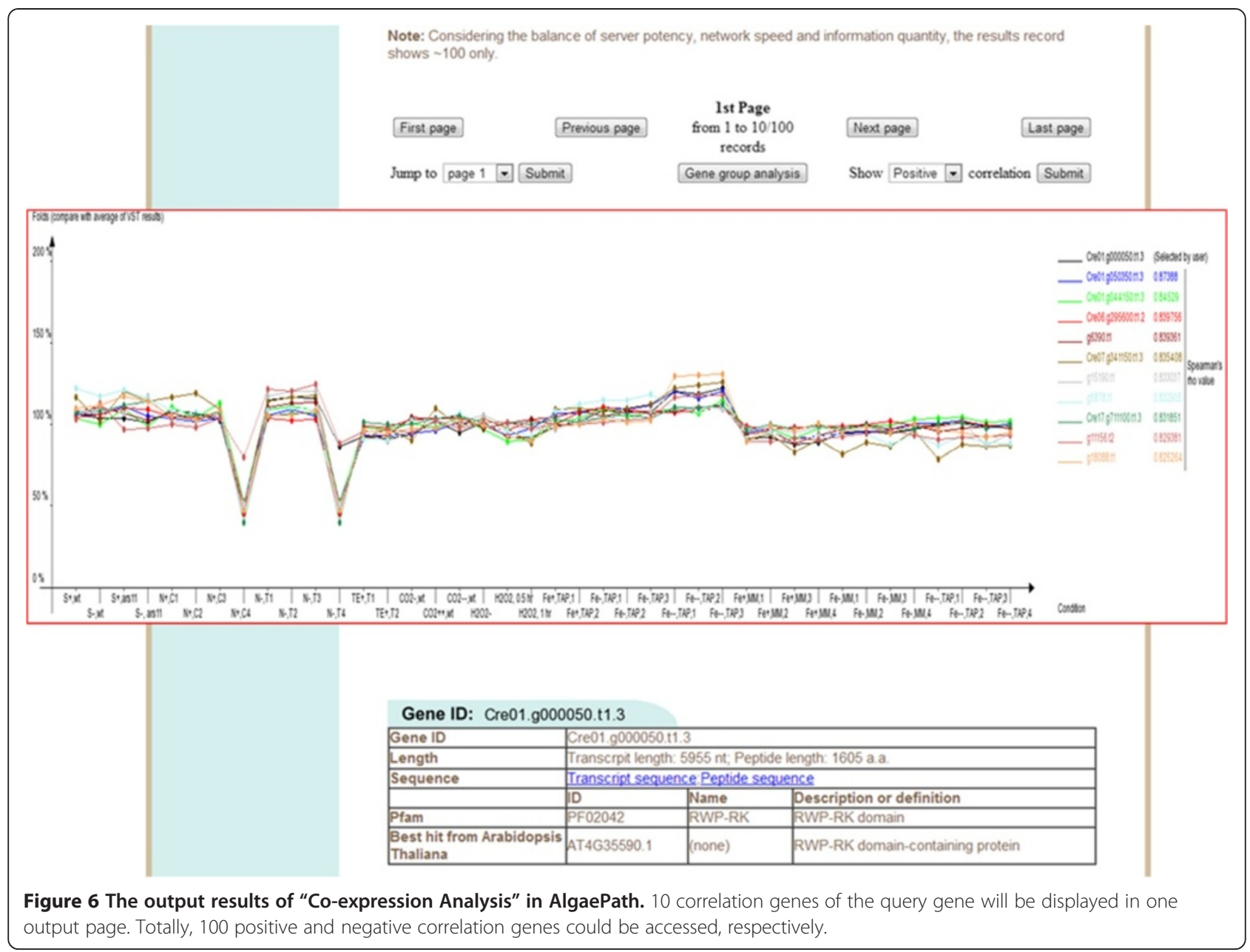

were significantly induced during sulfur starvation (e.g., arylsulfatase (ARS), ATP-sulfurylase, sulfate transporter, and sulfite reductase) [35]. These genes were validated using the AlgaePath system because the output results demonstrated that the transcript abundance levels increased obviously under sulfur depletion. Based on the AlgaePath data, the folds change between the sulfur starvation treatment and control samples, ARS (Cre16. g671350.t1.2), ATP-sulfurylase (Cre02.g107450.t1.2), sulfate transporter (Cre17.g723350.t1.2), and sulfite reductase (Cre09.g410750.t1.2) genes was found to be up-regulated by more than 2 folds (P-value <0.01) (Figure 7). These results suggest that AlgaePath can accurately represent phenomena reported in previous studies and can be used to identify the responses of some important genes to environmental stresses.

\section{Evaluation of AlgaePath and future perspectives}

The Algal Functional Annotation Tool (AFAT) integrates many algae databases for use in algae research [22]. AFAT has large-scale analysis functions, including pathway enrichment analysis and differential expression analysis; however, despite its capability for processing highthroughput or microarray results, AFAT has a number of limitations. For example, most of the query tools in AFAT require specific gene IDs from specific data sets (Augustus u10.2, Augustus v5.0, JGI v3.0, JGI v4.0) and no gene symbols or sequences are allowed as input. Further, transcript abundance levels and pathways in AFAT are presented on separate result pages, which makes it difficult for users to evaluate expression patterns under various conditions in a pathway. Therefore, in this work a user-friendly, web-based database for algae was developed. The results of a comparison between the AFAT and AlgaePath characteristics are shown in Table 1. A number of different query types are available in AlgaePath, and the transcript abundance data under different conditions are displayed simultaneously in a pathway map. Broadly, AlgaePath can be used to retrieve a cellular response in a pathway under a specific condition. Identifying differentially expressed genes between various samples is crucial to elucidating biological response mechanisms under a particular condition. Notably, the AlgaePath system helps users find critical genes related to cellular responses 


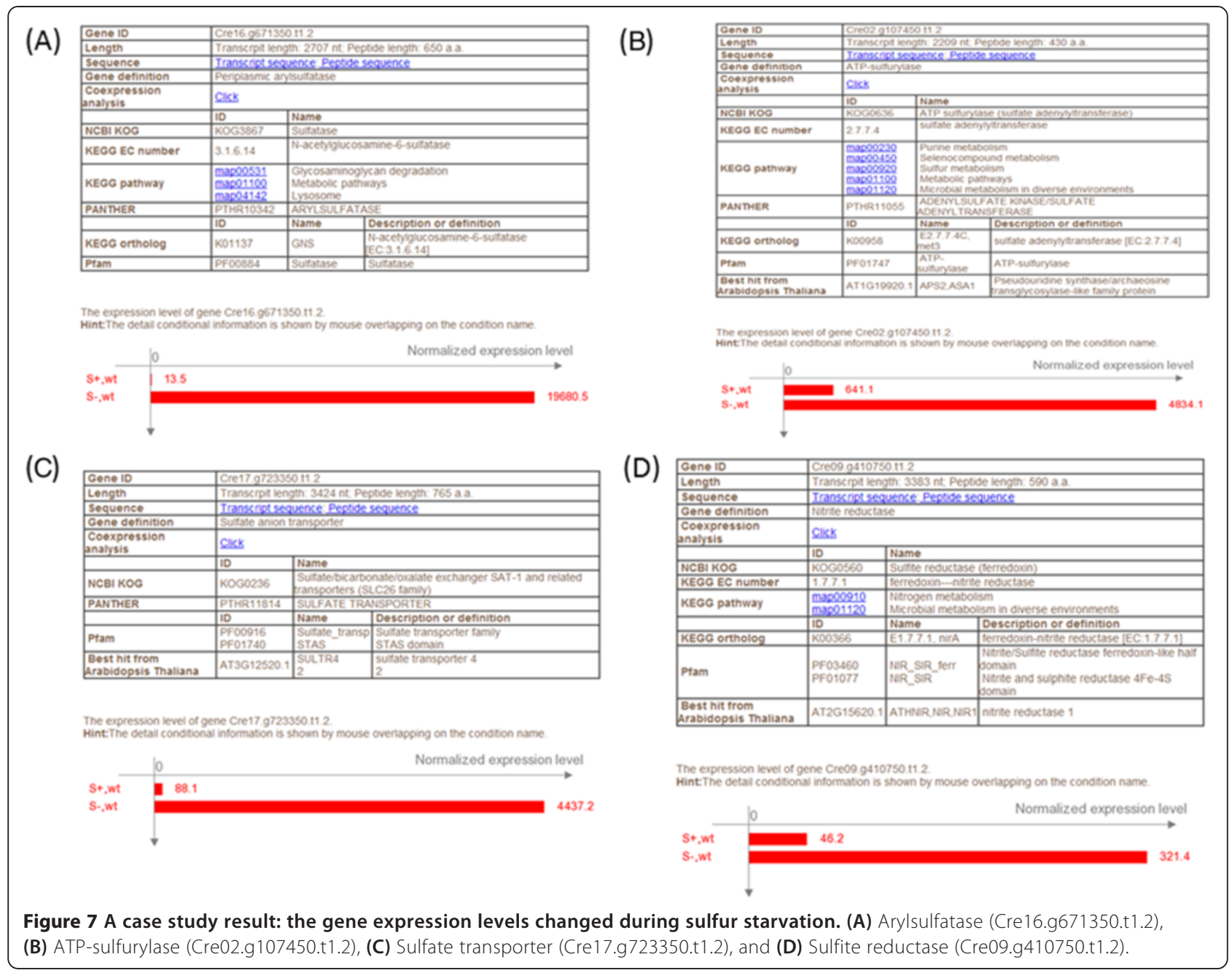

relatively easily. The ability to search for similar expression profiles is usually necessary to investigate the function of a novel gene. Both AFAT and AlgaePath can be used to identify co-expressed genes; however, in AFAT, determining gene functions based only on a gene ID can be rather challenging. To retrieve gene functions in AFAT, users have to access JGI (http://genome.jgi-psf.org/) online by clicking each ID number. On the other hand, in AlgaePath it is relatively easy to study genes that are positively and negatively correlated with the query gene,

Table 1 The comparison between algal functional annotation tool (AFAT) [22] and AlgaePath

\begin{tabular}{|c|c|c|}
\hline Content & AFAT & AlgaePath \\
\hline \multirow[t]{2}{*}{ Species } & Chlamydomonas reinhardtii & Chlamydomonas reinhardtii \\
\hline & Chlorella NC64A & Neodesmus sp. UTEX 2219-4 \\
\hline Search interface & Identifiers ID and keyword & $\begin{array}{l}\text { Gene symbol, Gene ID from various database, keyword, } \\
\text { DNA/protein sequences }\end{array}$ \\
\hline Pathways information of each transcript & Yes, only pathway map (out link to KEGG) & $\begin{array}{l}\text { Yes, combine pathway map with transcript abundance } \\
\text { profiles under various conditions }\end{array}$ \\
\hline Gene group analysis (Pathway enrichment) & $\begin{array}{l}\text { Yes, only mark genes in a pathway map } \\
\text { (out link to KEGG) }\end{array}$ & $\begin{array}{l}\text { Yes, not only mark genes in a pathway map but with } \\
\text { transcript abundance profiles under various conditions }\end{array}$ \\
\hline Differentially expression genes & No & $\begin{array}{l}\text { Yes, easily identify differentially expressed genes between } \\
\text { two samples }\end{array}$ \\
\hline Expression similar search & $\begin{array}{l}\text { Yes, only provide identifier ID in the } \\
\text { expression map }\end{array}$ & Yes, provide detail information of co-expression genes \\
\hline Pathway map with gene expression profiles & No & Yes \\
\hline
\end{tabular}


and all gene lists and information are downloadable from the web site.

A major advantage of AlgaePath over AFAT lies in its ability to integrate transcript abundance and pathway maps; however, although the genome sequence of $C$. reinhardtii was completed in 2007, the functions of many of the genes are still unknown [36]. In addition, some of the algal gene information in AlgaePath is limited; therefore, as more high-throughput experimental datasets become available it will be essential to continuously update the high-throughput data in AlgaePath. Although single datasets can be used to find interesting phenomena and to solve some related problems, the integration of many datasets can help increase the ability of researchers to identity variations in cellular responses under various conditions. Therefore, an improved normalization method needs to be developed to normalize many datasets from various platforms, including microarray, and Roche 454 or Illumina.

\section{Conclusions}

The emergence of whole transcriptome and genome research has made an integrative database for displaying cellular response essential. Algae are important non-model plants that have many research applications, including higher species diversity, sources of biofuel, adsorption of heavy metals and, following processing, health supplements. AlgaePath is a web-based database that comprehensively integrates C. reinhardtii and Neodesmus sp. UTEX 2219-4 gene information, biological pathways, and transcript abundance profiles from various databases. AlgaePath provides an effective interface for users interested in obtaining further insights into the transcript response mechanisms in different metabolic pathways under various conditions. Moreover, the Gene Group Analysis and Co-expression Analysis tools in AlgaePath can be used to detect similar expression transcripts of a target gene and enrichment pathways (functions) in a gene group. The results obtained using AlgaePath will provide a valuable reference for future efforts to elucidate critical mechanisms by mining highthroughput data. Importantly, the AlgaePath database is a significant contribution to algae research.

\section{Availability and requirements}

The AlgaePath database is publicly available at http:// AlgaePath.itps.ncku.edu.tw.

\section{Additional file}

Additional file 1: Table S1. The comparison groups for differentially expressed genes (DEGs) identification in AlgaePath.

\section{Competing interests}

The authors declare that they have no competing interests.

\section{Authors' contributions}

WCC conceived the analysis and main features of the tool, and wrote the manuscript. $\mathrm{CHC}$ designed the user interface. $\mathrm{HQZ}$ and $\mathrm{YFCH}$ wrote and tested the code, constructed AlgaePath database. BKJH help to normalize RNA-seq raw data by Deseq R package. TLL and CNNC provided access to the expression data and tested the tool. All authors read, edited and approved the final manuscript.

\section{Acknowledgments}

This research was supported by a grant from National Science Council of the Republic of China for financially supporting this research under Contract NSC 99-2628-B-006 -016 -MY3and NSC 102-2313-B-006-004. Ted Knoy is appreciated for his editorial assistance.

\section{Author details}

${ }^{1}$ Institute of Tropical Plant Sciences, National Cheng Kung University, Tainan 70101, Taiwan. ${ }^{2}$ Institute of Bioinformatics and Biosignal Transduction, National Cheng Kung University, Tainan 70101, Taiwan. ${ }^{3}$ Institute of Marine Biology, National Sun Yat-sen University, Kaohsiung 80424, Taiwan.

${ }^{4}$ Yourgene Bioscience Company Ltd, New Taipei City 23863, Taiwan.

Received: 4 December 2013 Accepted: 26 February 2014

Published: 14 March 2014

\section{References}

1. McKendry P: Energy production from biomass (part 1): overview of biomass. Bioresour Technol 2002, 83(1):37-46.

2. McKendry P: Energy production from biomass (part 2): conversion technologies. Bioresour Technol 2002, 83(1):47-54.

3. Martin MA: First generation biofuels compete. N Biotechnol 2010, 27(5):596-608.

4. Fan J, Yan C, Andre C, Shanklin J, Schwender J, Xu C: Oil accumulation is controlled by carbon precursor supply for fatty acid synthesis in Chlamydomonas reinhardtii. Plant Cell Physio/ 2012, 53(8):1380-1390.

5. Singh J, Gu S: Commercialization potential of microalgae for biofuels production. Renew Sust Energ Rev 2010, 14(9):2596-2610.

6. Lommer M, Specht M, Roy AS, Kraemer L, Andreson R, Gutowska MA, Wolf J, Bergner SV, Schilhabel MB, Klostermeier UC, Beiko RG, Rosenstiel P, Hippler M, Laroche J: Genome and low-iron response of an oceanic diatom adapted to chronic iron limitation. Genome Biol 2012, 13(7):R66.

7. Çetinkaya Dönmez G, Aksu Z, Öztürk A, Kutsal T: A comparative study on heavy metal biosorption characteristics of some algae. Process Biochem 1999, 34(9):885-892.

8. Roy D, Greenlaw PN, Shane BS: Adsorption of heavy metals by green algae and ground rice hulls. J Environ Sci Health Part A 1993, 28(1):37-50.

9. Gupta V, Shrivastava A, Jain N: Biosorption of Chromium (VI) from Aqueous solutions by green algae spirogyra species. Water Res 2001, 35(17):4079-4085.

10. Katz ME, Wright JD, Miller KG, Cramer BS, Fennel K, Falkowski PG: Biological overprint of the geological carbon cycle. Mar Geol 2005, 217(3):323-338.

11. Longhurst AR: Role of the marine biosphere in the global carbon cycle. Limnol Oceanogr 1991, 36(8):1507-1526.

12. Wang ST, Pan YY, Liu CC, Chuang LT, Chen CNN: Characterization of a green microalga UTEX 2219-4: effects of photosynthesis and osmotic stress on oil body formation. Bot Stud 2011, 52(3):305-312.

13. Boyle NR, Page MD, Liu B, Blaby IK, Casero D, Kropat J, Cokus SJ, Hong-Hermesdorf A, Shaw J, Karpowicz SJ, Gallaher SD, Johnson S, Benning C, Pellegrini M, Grossman A, Merchant SS: Three acyltransferases and nitrogen-responsive regulator are implicated in nitrogen starvation-induced triacylglycerol accumulation in Chlamydomonas. J Bio/ Chem 2012, 287(19):15811-15825.

14. Fang W, Si Y, Douglass S, Casero D, Merchant SS, Pellegrini M, Ladunga I, Liu P, Spalding MH: Transcriptome-wide changes in Chlamydomonas reinhardtii gene expression regulated by carbon dioxide and the $\mathrm{CO} 2$-concentrating mechanism regulator CIA5/CCM1. Plant Cell 2012, 24(5):1876-1893.

15. Fischer BB, Ledford HK, Wakao S, Huang SG, Casero D, Pellegrini M, Merchant SS, Koller A, Eggen Rl, Niyogi KK: Singlet oxygen resistant 1 links reactive electrophile signaling to singlet oxygen acclimation in Chlamydomonas reinhardtii. Proc Natl Acad Sci U S A 2012, 109(20):E1302-E1311.

16. Gonzalez-Ballester D, Casero D, Cokus S, Pellegrini M, Merchant SS, Grossman AR: RNA-seq analysis of sulfur-deprived Chlamydomonas cells reveals aspects of acclimation critical for cell survival. Plant Cell 2010, 22(6):2058-2084 
17. Miller R, Wu G, Deshpande RR, Vieler A, Gartner K, Li X, Moellering ER, Zauner S, Cornish AJ, Liu B, Bullard B, Sears BB, Kuo MH, Hegg EL, Shachar-Hill Y, Shiu SH, Benning $C$ : Changes in transcript abundance in Chlamydomonas reinhardtii following nitrogen deprivation predict diversion of metabolism. Plant Physiol 2010, 154(4):1737-1752.

18. Urzica El, Adler LN, Page MD, Linster CL, Arbing MA, Casero D, Pellegrini M, Merchant SS, Clarke SG: Impact of oxidative stress on ascorbate biosynthesis in Chlamydomonas via regulation of the VTC2 gene encoding a GDP-Lgalactose phosphorylase. J Biol Chem 2012, 287(17):14234-14245.

19. Hruz T, Laule O, Szabo G, Wessendorp F, Bleuler S, Oertle L, Widmayer $P$, Gruissem W, Zimmermann P: Genevestigator v3: a reference expression database for the meta-analysis of transcriptomes. Adv. Bioinform 2008, 2008:420747.

20. Patel RV, Nahal HK, Breit R, Provart NJ: BAR expressolog identification: expression profile similarity ranking of homologous genes in plant species. Plant J cCell Mole Biol 2012, 71(6):1038-1050.

21. Latendresse M, Paley S, Karp PD: Browsing metabolic and regulatory networks with BioCyc. Methods Mol Biol 2012, 804:197-216.

22. Lopez D, Casero D, Cokus SJ, Merchant SS, Pellegrini M: Algal functional annotation tool: a web-based analysis suite to functionally interpret large gene lists using integrated annotation and expression data. BMC Bioinform 2011, 12:282.

23. Goodstein DM, Shu S, Howson R, Neupane R, Hayes RD, Fazo J, Mitros T, Dirks W, Hellsten U, Putnam N, Rokhsar DS: Phytozome: a comparative platform for green plant genomics. Nucleic Acids Res 2012, 40(Database issue):D1178-D1186.

24. Punta M, Coggill PC, Eberhardt RY, Mistry J, Tate J, Boursnell C, Pang N Forslund K, Ceric G, Clements J, Heger A, Holm L, Sonnhammer EL, Eddy SR, Bateman A, Finn RD: The Pfam protein families database. Nucleic Acids Res 2012, 40(Database issue):D290-D301.

25. Mi H, Muruganujan A, Thomas PD: PANTHER in 2013: modeling the evolution of gene function, and other gene attributes, in the context of phylogenetic trees. Nucleic Acids Res 2013, 41(Database issue):D377-D386.

26. Tatusov RL, Fedorova ND, Jackson JD, Jacobs AR, Kiryutin B, Koonin EV, Krylov DM, Mazumder R, Mekhedov SL, Nikolskaya AN: The COG database: an updated version includes eukaryotes. BMC Bioinform 2003, 4(1):41.

27. Langmead B, Salzberg SL: Fast gapped-read alignment with Bowtie 2. Nat Methods 2012, 9(4):357-359.

28. Kent WJ: BLAT-the BLAST-like alignment tool. Genome Res 2002, 12(4):656-664.

29. Hu Q, Sommerfeld M, Jarvis E, Ghirardi M, Posewitz M, Seibert M, Darzins A: Microalgal triacylglycerols as feedstocks for biofuel production: perspectives and advances. Plant J Cell Mole Biol 2008, 54(4):621-639.

30. Kanehisa M, Goto S: KEGG: kyoto encyclopedia of genes and genomes. Nucleic Acids Res 2000, 28(1):27-30.

31. Huttenhower C, Haley EM, Hibbs MA, Dumeaux V, Barrett DR, Coller HA, Troyanskaya OG: Exploring the human genome with functional maps. Genome Res 2009, 19(6):1093-1106.

32. Rosenberg JN, Oyler GA, Wilkinson L, Betenbaugh MJ: A green light for engineered algae: redirecting metabolism to fuel a biotechnology revolution. Curr Opin Biol 2008, 19(5):430-436.

33. Rismani-Yazdi H, Haznedaroglu BZ, Bibby K, Peccia J: Transcriptome sequencing and annotation of the microalgae Dunaliella tertiolecta: pathway description and gene discovery for production of nextgeneration biofuels. BMC Genomics 2011, 12:148.

34. Wang ZT, Ullrich N, Joo S, Waffenschmidt S, Goodenough U: Algal lipid bodies: stress induction, purification, and biochemical characterization in wild-type and starchless Chlamydomonas reinhardtii. Eukaryot Cell 2009, 8(12):1856-1868

35. Zhang Z, Shrager J, Jain M, Chang CW, Vallon O, Grossman AR: Insights into the survival of Chlamydomonas reinhardtii during sulfur starvation based on microarray analysis of gene expression. Eukaryot Cell 2004, 3(5):1331-1348.

36. Merchant SS, Prochnik SE, Vallon O, Harris EH, Karpowicz SJ, Witman GB, Terry A, Salamov A, Fritz-Laylin LK, Marechal-Drouard L, et al: The Chlamydomonas genome reveals the evolution of key animal and plant functions. Science 2007, 318(5848):245-250.

doi:10.1186/1471-2164-15-196

Cite this article as: Zheng et al:: AlgaePath: comprehensive analysis of metabolic pathways using transcript abundance data from next-generation sequencing in green algae. BMC Genomics 2014 15:196.

\section{Submit your next manuscript to BioMed Central and take full advantage of:}

- Convenient online submission

- Thorough peer review

- No space constraints or color figure charges

- Immediate publication on acceptance

- Inclusion in PubMed, CAS, Scopus and Google Scholar

- Research which is freely available for redistribution 